\title{
Author Correction: Treating medical data as a durable asset
}

Amalio Telenti (D) and Xiaoqian Jiang (1)

Correction to: Nature Genetics https://doi.org/10.1038/s41588-020-0698-y, published online 14 September 2020.

In the version of this article initially published, the authors made a broad statement regarding the business models of commercial enterprises in genomics ('In several conspicuous examples, commercial enterprises in genomics (for example, 23andMe, AncestryDNA, MyHeritageDNA and others) have business models that depend on exploring and reselling their customers' genetic data and phenotypic information'). Practices across companies differ, thus affecting the way data are managed. On this basis, the authors have modified the original text to 'Commercial enterprises in genomics (for example, 23andMe, AncestryDNA, MyHeritageDNA and others) build value by using customers' data-users should attentively read the terms and conditions and privacy policies to assess each company's use of genetic data and phenotypic information.' The text has been corrected in the HTML and PDF versions of the article.

Published online: 8 October 2020

https://doi.org/10.1038/s41588-020-00734-6

๑) The Author(s), under exclusive licence to Springer Nature America, Inc. 2020

\section{Publisher Correction: A genome-wide search for asthma susceptibility loci in ethnically diverse populations}

The Collaborative Study on the Genetics of Asthma (CSGA)

Correction to: Nature Genetics https://doi.org/10.1038/ng0497-389, published online 1 April 1997.

Owing to technical issues, the author name is missing from the HTML version of this article; it should be "The Collaborative Study on the Genetics of Asthma (CSGA)'. The author name in the PDF is correct. The contact for the CSGA is Carole Ober at The University of Chicago, Chicago, Illinois, USA. A full list of the individual authors and their affiliations appears in https://www.nature.com/articles/ ng0497-389.pdf.

Published online: 22 October 2020

https://doi.org/10.1038/s41588-020-0650-1

(c) The Author(s), under exclusive licence to Springer Nature America, Inc. 2020 